\title{
Tumeur du sac vitellin du testicule au stade Illc métastatique : à propos d'un cas
}

\author{
Mohamed Zizi, MD; Imad Ziouziou, MD;* Souhail El Yacoubi, MD; ${ }^{*}$ Mouna Khmou, MD; ${ }^{+}$Ahmed Jahid, MD; \\ Najat Mahassini, MD; ${ }^{\dagger}$ Tariq Karmouni, MD; Khalid El Khader, MD; ${ }^{*}$ Abdellatif Koutani, MD; \\ Ahmed Iben Attya Andaloussi, MD*
}

'Service d'urologie B, CHU Ibn Sina, Faculté de médecine et pharmacie de Rabat, Université Mohamed V souissi, Rabat, Maroc; 'Service d'anatomie pathologique, CHU lbn Sina, Rabat, Maroc

Cite as: Can Urol Assoc J 2014;8(9-10):e663-5. http://dx.doi.org/10.5489/cuai.676 Published online September 9, 2014.

\section{Résumé}

Les tumeurs du sac vitellin du testicule sont rares chez l'adulte. Ces tumeurs se caractérisent par un mauvais pronostic à un stade métastatique avancé. Cependant, nous rapportons, dans le présent article, le cas clinique d'un adulte de 32 ans qui présentait une tumeur du sac vitellin du testicule au stade IIlc métastatique. Ce patient a subi une orchidectomie haute, accompagnée de quatre cycles de chimiothérapie à base de bléomycine, d'étoposide et de cisplatine. II a répondu complètement au traitement, moyennant un recul de deux ans.

\section{Introduction}

La tumeur du sac vitellin est différenciée dans le sens extraembryonnaire et reproduit des structures évocatrices du sac vitellin de l'homme. Elle se définit, sur le plan histologique, par la présence de cellules tumorales organisées autour des vaisseaux caractéristiques formant les corps de SchillerDuval. Ces tumeurs du testicule sont rares chez l'adulte, mais ont un mauvais pronostic à leur stade métastatique avancé.

Nous présentons, dans cet article, le cas clinique d'un patient atteint d'une tumeur du sac vitellin du testicule au stade IIIc métastatique. Ce patient a répondu complètement au traitement.

\section{Observation}

Monsieur A. T., âgé de 32 ans, a consulté pour une grosse bourse droite évoluant depuis 4 ans. Il ne présentait pas d'antécédents de cancer. L'examen clinique a mis en évidence une masse testiculaire droite de $8 \mathrm{~cm}$, dure et sensible, sans signe inflammatoire. Le testicule controlatéral était normal. Les aires ganglionnaires étaient libres. L'échographie scrotale a objectivé un testicule droit de taille amplifiée (70 mm/50 mm), hétérogène, aux contours irréguliers, présentant de fines calcifications ayant été épargnées. L'épididyme n'était pas visible. La morphologie du testicule controlatéral était normale. Le dosage des marqueurs tumoraux a indiqué une élévation de la lacticodéshydrogénase (LDH) à 1158 UI/L (la normale s'établissant à 4,5). L'hormone chorionique gonadotrophique (hCG) totale s'établissait à un taux normal de 1,2 mUl/mL, de même que l'alpha-fœtoprotéine, qui s'élevait à 5,21 ng/mL.

Le diagnostic d'une tumeur testiculaire a été évoqué. Une orchidectomie par voie inguinale avec ligature première du cordon spermatique a été réalisée. L'examen anatomopathologique de la pièce opératoire a orienté le diagnostic en faveur d'une tumeur du sac vitellin (figure 1). Le taux de LDH a diminué en mode postopératoire à $753 \mathrm{UI} / \mathrm{L}$. L'imagerie thoraco-abdomino-pelvienne a objectivé des localisations secondaires au poumon et au foie, ainsi que des adénopathies rétropéritonéales mesurant $43 \mathrm{~mm} / 35 \mathrm{~mm}$ (figure 2, figure 3). La tumeur a été classée PT1 N2 M1b S2, au stade IIIc.

Le patient a reçu une chimiothérapie à raison de quatre cycles de BEP (bléomycine, étoposide et cisplatine). À la fin du traitement, son taux de LDH s'établissait à 294 UI/L, et I'imagerie thoraco-abdomino-pelvienne objectivait la persistance des images nodulaires liées aux 2 hémichamps pulmonaires, un foie normal sans lésion focale ainsi qu'un magma d'adénopathies inter-aortico-caves mesurant $13 \mathrm{~mm} / 11 \mathrm{~mm}$ et latéro-aortiques gauches. Trois mois plus tard, un examen biologique a indiqué une normalisation de la LDH. Cependant, à la tomodensitométrie, les nodules pulmonaires et rétropéritonéaux $\mathrm{n}^{\prime}$ avaient pas diminué de taille. Par la suite, le patient a fait l'objet d'un suivi clinique, biologique (marqueurs) et radiologique (imagerie thoracoabdomino-pelvienne) tous les trois mois. Six mois plus tard, 


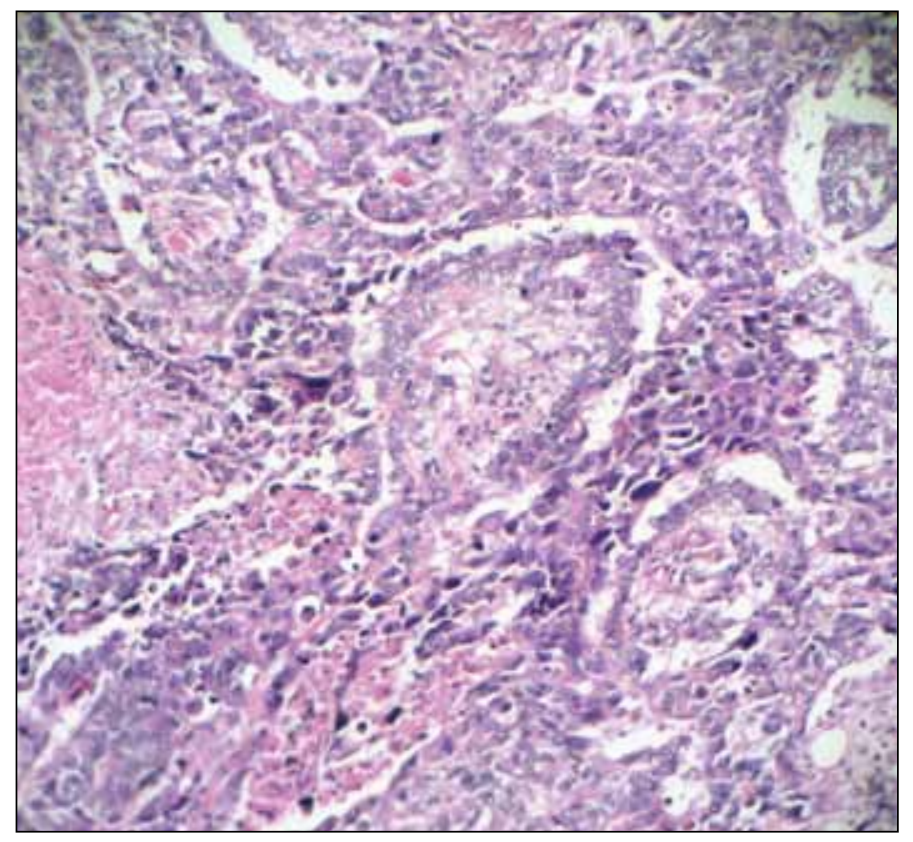

Fig. 1. Infiltrat tumoral sous forme de pseudo-papilles et corps de SchillerDuval caractéristiques (hémalun-éosine ×40).

les résultats étaient normaux à l'examen clinique, de même que les marqueurs (LDH à $179 \mathrm{UI} / \mathrm{L}$ ). La tomodensitométrie indiquait une réponse complète au traitement : absence de lésions évolutives avec remaniements séquellaires interaortico-caves. Un an plus tard, l'examen de suivi était en faveur $d^{\prime}$ une réponse complète au traitement, et la masse rétropéritonéale avait disparu (figure 4).

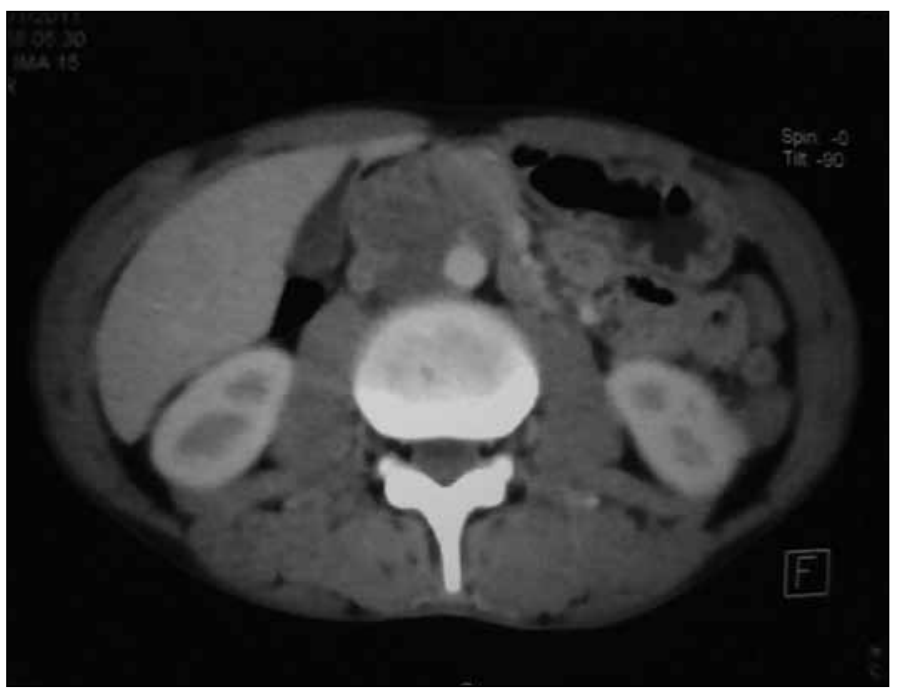

Fig. 3. Tomodensitométrie coupe axiale abdominale montrant une masse rétropéritonéale importante.

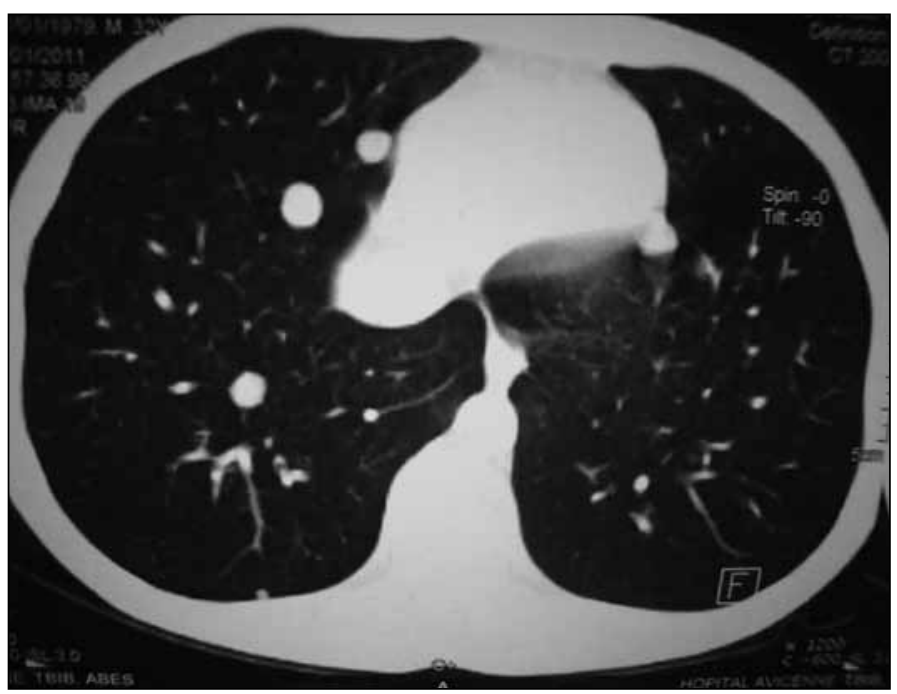

Fig. 2. Tomodensitométrie coupe thoracique montrant des métastases pulmonaires.

\section{Discussion}

La tumeur du sac vitellin est un type histologique rare de tumeur du testicule.

Elle représente $72 \%$ des tumeurs du testicule chez $I^{\prime}$ enfant de 1 à 2 ans $^{1,2}$. Elle s'associe souvent à d'autres contingents cellulaires chez l'adulte avec une fréquence qui a tendance à augmenter (44\%), vu son dépistage systématique par l'emploi de marqueurs sériques $(\alpha \mathrm{FP})$ et tissulaires ${ }^{2,3}$.

Dans le cas qui nous occupe, la tumeur du sac vitellin était de forme pure. Il s'agissait d'une tumeur différenciée dans le sens extra-embryonnaire. Ce type de tumeur reproduit des structures évocatrices du sac vitellin de l'homme ou du sinus endodermique du rat, et exprime de façon spé-

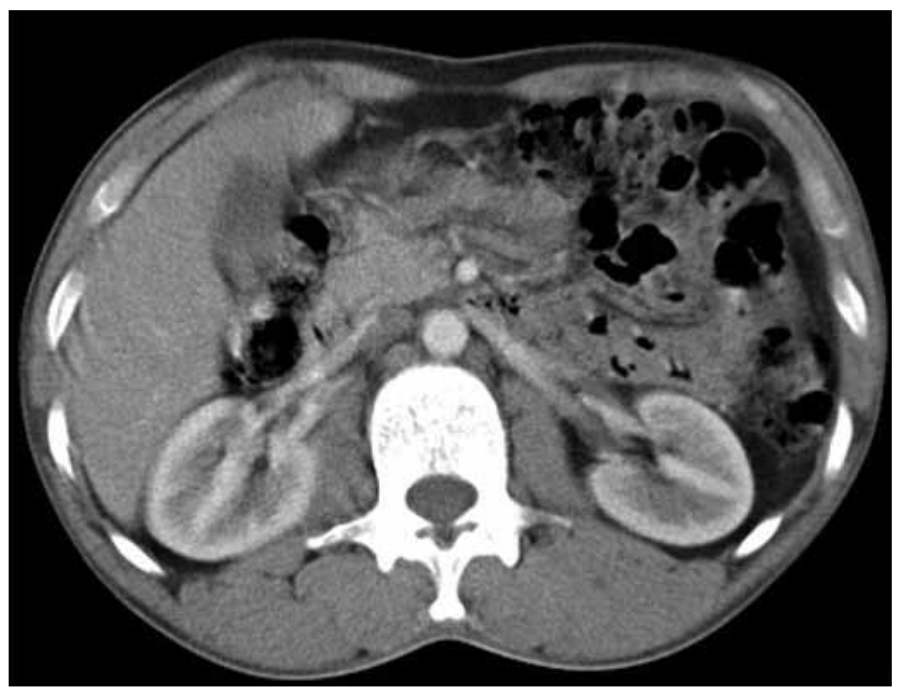

Fig. 4. Tomodensitométrie après un an montrant l'absence de masse résiduelle. 
Tumeur du sac vitellin du testicule au stade IIIc métastatique

cifique et quasi-constante I'alphafotoprotéine $(\alpha \mathrm{FP})^{4,5}$. On observe une augmentation de I' $\alpha \mathrm{FP}$ sérique chez plus de $90 \%$ des patients ${ }^{3}$. Notre cas avait la particularité d'être une tumeur du sac vitellin $\alpha \mathrm{FP}$ négative. Les tumeurs du sac vitellin sont habituellement diagnostiquées au moyen $d^{\prime}$ 'une coloration à l'hématoxyline et à l'éosine des coupes tissulaires. Cependant, elles peuvent mimer d'autres types de tumeurs germinales et non germinales, surtout dans les sites métastatiques et extragonadiques. Pour cette raison, les marqueurs immunohistochimiques sont parfois utilisés pour faciliter et étayer le diagnostic ${ }^{3}$.

Les marqueurs immunohistochimiques classiques des tumeurs du sac vitellin comprennent notamment l' $\alpha \mathrm{FP}$, la phosphatase alcaline placentaire (PLAP) et le glypican-3. Ces marqueurs manquent toutefois de sensibilité et de spécificité, particulièrement dans les sites extragonadiques. Récemment, la protéine sal-like 4 (SALL4) a été introduite à titre de nouveau marqueur des tumeurs du sac vitellin. Elle est plus sensible que I' $\alpha \mathrm{FP}$, le PLAP et le glypican-3 pour les sites gonadiques et extragonadiques ${ }^{3,6-9}$. Le diagnostic reste de nature histologique après orchidectomie par voie inguinale et met en évidence les corps de Schiller-Duval ${ }^{3}$. La chimiothérapie à raison de quatre cycles de BEP est le traitement de référence pour les tumeurs germinales non séminomateuses métastatiques de stade III $^{10}$.

Dans le cas de notre patient, aucune chirurgie des masses résiduelles rétropéritonéales n'a été réalisée après la chimiothérapie, à cause de l'absence de normalisation de la LDH. Cette normalisation est obligatoire pour procéder à cette chirurgie ${ }^{11}$. Le pronostic était mauvais pour notre patient selon la Classification pronostique IGCCCG (International Germ Cell Cancer Collaborative Group) des stades métastatiques, du fait de la présence de métastases extrapulmonaires (hépatiques) et d'un taux de $\mathrm{LDH}$ supérieur à $10 \mathrm{UI} / \mathrm{IL}^{12}$. Selon l'étude à répartition aléatoire réalisée par Motzer et coll., l'évolution des tumeurs germinales - qui représentent un risque intermédiaire à important - est marquée par un taux de réponse complète de $48 \%$ au bout de 1 an, après 4 cycles de chimiothérapie à base de bléomycine, d'étoposide et de cisplatine ${ }^{13}$. Chez notre patient, les métastases pulmonaires et hépatiques, de même que les masses rétropéritonéales, ont complètement disparu moyennant un recul de deux ans.

\section{Conclusion}

Dans leur forme pure, les tumeurs du sac vitellin sont des tumeurs rares du testicule chez l'adulte. Des études ultéri- eures sont toutefois nécessaires pour mieux en comprendre les aspects thérapeutiques et évolutifs.

Competing interests: Dr. Zizi. Dr. Ziouziou, Dr. El Yacoubi, Dr. Khmou, Dr. Jahid, Dr. Mahassini, Dr. Karmouni, Dr. El Khader, Dr. Koutani and Dr. Andaloussi all declare no competing financial or personal interests.

This paper has been peer-reviewed.

\section{Références}

1. Green DM. The diagnosis and treatment of yolk sac tumor in infants and children. Cancer Treat Rev 1983;10:265. http://dx.doi.org/10.1016/0305-7372(83)90014-2

2. Debbagh A, Bennani S, Jouhadi H, et al. Tumeur du sac vitellin. À propos d'un cas. Ann Urol 2001;35:3568. http://dx.doi.org/10.1016/S0003-4401 (01)00060-2

3. Cao D, Humphrey PA. Yolk sac tumor of the testis. J Urol 2011;186:1475-6. http://dx.doi. org/10.1016/i.juro.2011.06.045

4. Perrin P, Bailly C, Berger-Dutrieux N, et al. Cancers du testicule. Cancers uro-génitaux. Paris, France,Flammarion: 308-37.

5. Berger N, Voigt JJ. Cancer du testicule. Anatomie pathologique. Rev Prat 1993;43:1717-20.

6. Cao D, Li J, Guo CC, et al. SALL4 is a novel diagnostic marker for testicular germ cell tumors. Am I Surg Pathol 2009;33:1065. http://dx.doi.org/10.1097/PAS.0b013e3181a13eef

7. Cao D, Humphrey PA, Allan RW, et al. SALL 4 is a novel sensitive and specific marker for metastatic germ cell tumors, with particular utility in detection of metastatic yolk sac tumors. Cancer 2009;115:2640. http://dx.doi.org/10.1002/cncr.24308

8. Wang F, Liu A, Peng Y, et al. Diagnostic utility of SALL4 in extragonadal yolk sac tumors: an immunohistochemical study of 59 cases with comparison to placental-like alkaline phosphatase, alpha-fetoprotein, and glypican-3. Am J Surg Pathol 2009;33:1529. http://dx.doi.org/10.1097/PAS.0b013e3181ad25d5

9. Cao D, Guo S, Allan RW, et al. SALL4 is a novel sensitive and specific marker of ovarian primitive germ cell tumors and is particularly useful in distinguishing yolk sac tumor from clear cell carcinoma. Am I Surg Pathol 2009;33:894. http://dx.doi.org/10.1097/PAS.0b013e318198177d

10. Durand X, Rigaud J, Avances C, et al. Soulié et les membres du CCAFU. Recommandations en onco-urologie 2010 : Tumeurs germinales du testicule. Prog Urol 2010;S297-S311. http://dx.doi.org/10.1016/ S1166-7087(10)70046-4

11. Kollmannsberger C, Daneshmand S, So A, et al. Management of disseminated nonseminomatous germ cell tumors with risk-based chemotherapy followed by response-guided postchemotherapy surgery. I Clin Oncol 2010;28:537-42. http://dx.doi.org/10.1200/JC0.2009.23.0755

12. International Germ Cell Consensus Classification : A prognostic factor-based staging system for metastatic germ cell cancers. International Germ Cell Cancer Collaborative Group. J Clin Oncol 1997;15:594-603.

13. Motzer RJ, Nichols CJ, Margolin KA, et al. Phase III randomized trial of conventional-dose chemotherapy with or without high-dose chemotherapy and autologous hematopoietic stem-cell rescue as first-line treatment for patients with poor-prognosis metastatic germ cell tumors. J Clin Oncol 2007;25:247-56. http://dx.doi.org/10.1200/JC0.2005.05.4528

Correspondence: Dr. Imad Ziouziou, Service d'urologie B, CHU lbn Sina, Faculté de médecine et pharmacie de Rabat, Université Mohamed V souissi, Rabat, Maroc; imadziouziou@hotmail.com 